\title{
Astronomical Units and Constants in a Relativistic Framework
}

\author{
N. Capitaine ${ }^{*}$ and B. Guinot*** \\ * Observatoire de Paris, DANOF/URA1125, 75014 Paris, France \\ * rue des Soupirs, 77590 Chartrettes, France
}

In 1991, IAU Resolution A4 introduced General Relativity as the theoretical background for defining celestial space-time reference sytems. It is now essential that units and constants used in dynamical astronomy be defined in the same framework, at least in a manner which is compatible with the minimum degree of approximation of the metrics given in Resolution A4.

This resolution states that astronomical constants and quantities should be expressed in SI units, but does not consider the use of astronomical units. We should first evaluate the usefulness of maintaining the system of astronomical units. If this system is kept, it must be defined in the spirit of Resolution A4. According to Huang T.-Y., Han C.-H., Yi Z.-H., Xu B.-X. (What is the astronomical unit of length?, to be published in Astron. Astrophys.), the astronomical units for time and length are units for proper quantities and are therefore proper quantities. We fully concur with this point of view. Astronomical units are used to establish the system of graduation of coordinates which appear in ephemerides: the graduation units are not, properly speaking astronomical units. Astronomical constants, expressed in SI or astronomical units, are also proper quantities.

Among the questions which arise from a relativistic definition of astronomical units, we mention the following ones.

1. Should the numerical value of the defining constant $k$ (Gaussian gravitational constant) be conserved? For example, a change of the definition of the astronomical units of length and time (the day) could keep unchanged the coordinates of existing ephemerides.

2. Is $k$ the best defining constant? The universal constant of gravitation $G\left(=k^{2}\right)$ seems more appropriate because it is a fundamental constant of physics, known by everyone, and because the geometrical interpretation of $k$ in General Relativity may be misleading. In astronomical units, numerically, $G=G M_{\text {Sun }}$.

3. Is $\tau_{\mathrm{A}}$ (light time in seconds for the astronomical unit of length) the best primary constant to link astronomical and SI units? A more logical choice would be to provide $G M_{\text {Sun }}$ in SI units, because it is a fundamental quantity for those who work in SI units. It provides the link with astronomical units, since $G M_{\text {Sun }}$ is known, by definition, in these units. Of course, $G M_{\text {Sun }}$ in SI can be derived from $\tau_{A}$, but in an indirect way, which introduces the astronomical unit of length where it is not needed.

Before defining a new IAU system of astronomical constants, such questions must be considered. Then the meaning of astronomical units, constants and quantities must be clearly explicited, and made available, not only to specialists, but also to other astronomers, engineers, students, in a detailed IAU resolution, which could serve as an unambiguous basis for all explanatory documents, e. g. textbooks. We suggest to create a working group to study these matters. 